\title{
Computer-mediated communication as a learning resource
}

\author{
E. McAteer, A. Tolmie*, C. Duffy' \& J. Corbett \\ Teaching and Learning Service, University of Glasgow; *Dept. of Psychology, University of \\ Strathclyde; ${ }^{\dagger}$ Dept. of Music; ${ }^{\ddagger}$ Dept. of English Language, University of Glasgow
}

\begin{abstract}
Evaluative studies of CMC can produce misleading or even contradictory results due to an (understandable) focus on how the characteristics of the medium affect usage, ignoring the dialectic between technology and culture, of mutual adaptation over time. CMC exchanges in Higher Education take place within a broad teaching and learning system, of which most participants already have extensive experience. This system provides the context within which participants make sense of, and adapt to, the use of on-line communications. Thus interpretations of the processes shaping exchanges and their outcomes have to take into account: who participants see themselves as communicating with and why, how this serves longer-term learning goals, and what past experience of engaging with both task and audience they have had; what kinds of exchange are facilitated, both by the medium itself and how the task is organised, and how such affordances are honed over time; and also, how these interactions impact on other aspects of teaching and on learning outcomes.
\end{abstract}

Keywords: Computer-mediated communication; Evaluation; Higher Education; Music; Language studies; On-line seminars

\section{Introduction}

The focus of this paper is on computer-mediated communication (CMC) in Higher Education (HE), and in particular on the evaluation of network resources for course-related student communication, set up with the specific aim of supporting learning.

As well as a general psychological research interest in computer-mediated communication systems, the immediate concern is whether such developments in teaching practice are effective, practically and pedagogically. CMC forms an increasingly large component of many distance education programmes, where it is portrayed as an ideal means of providing opportunities for group

This paper arises from a contribution to an Anglo-Italian bilateral seminar funded by British Council/MURST which was held in Trieste, November, 1996. A version has been translated into Italian and published in Tecnologie Didattiche 12, Communicazione Mediata dal Computer, Primavera 1997.

Correspondence: Erica McAteer, Teaching and Learning Service, University of Glasgow, Robert Clark Centre, 66 Oakfield Ave., Glasgow G12 8LS Email: e.mcateer@udcf.gla.ac.uk 
discussion, tutor-student interaction and collaborative tasks. It is also becoming increasingly common in 'traditional' HE classes, where a growing number of teachers are persuaded that it can be used to generate communication of greater value for learning than standard classroom forms of interaction, as well as providing a forum space for students who may not have sufficiently common timetables to meet for course related discussions (Kaye, 1989).

The central questions to be addressed, from the twin perspectives of communication research and the evaluation of educational practice, are "How do CMC resources actually get used in practice, with what impact, and why?" - recognising that there is a diversity of systems and tasks. The focus here is deliberately placed as much on process as on outcome. In part this is a matter of pragmatics: evaluation of outcome alone might indicate if a resource is useful, but how and why it achieves that usefulness will provide a more powerful basis for further refinement and application in other contexts. The emphasis on process has a fundamental importance because of the complexity of the potential influences on CMC usage. Two case examples relating to the usage of email seminars demonstrate the significance of this last point.

\section{Case 1: Scottish Language Studies}

The Department of Scottish Language at the University of Glasgow makes good use of student computing labs, and is well resourced for text analysis, with an expanding amount of otherwise fairly inaccessible Scots texts from different periods available for study. Text analysis is an important component of the undergraduate degree, and the 'Stylistics of Scottish Literature' course requires third-year students to work through a variety of exercises to develop their skills with weekly lectures accompanying a series of computer-supported workshops. On-line seminars were already in place or under consideration in other courses in the department and it seemed a natural development to bring the two components together, with the intention of using the forum space to develop themes from the lectures, identify suitable texts for analysis and discuss technique.

In 1995/96 there were 30 students involved in the class. All were familiar with computers, using them fairly extensively for word processing, and having previous experience with 'drill and practice' exercises and basic text analysis routines. None had direct experience of email seminars, on-line forums or much familiarity with Internet resources. The students knew each other well, having worked through the Scottish Literature components of their first and second undergraduate years as a self-selected group. They also knew the tutor well a different situation from that obtaining with larger classes.

Suggestions for sample text searches were emailed to students by the class tutor on a regular basis and their task was simply to discuss each text raising questions relating to its analysis and generating possible solutions for testing at the week's on-line workshop. It was hoped that this would help students to build up skills in textual description, and to generate a shared vocabulary which 
would facilitate further discussion. The dialogue was asynchronous, with individual students using the computing lab resources in their own time out of class sessions, and although all contributions were delivered to all students in the group, the actual sender of each message was anonymous. There was little other structure provided, since the class tutor was not involved in the discussion, and students were not instructed on when or how often they should read or contribute to any seminar.

In the event, there was considerable usage of the resource, but only by a limited number of students. Since no record was kept of actual activity on-line, it is not possible to comment on the specific content or quality of the exchanges that took place. However, student feedback was positive amongst those who used the resource, indicating that it had certainly been perceived to be of benefit. The ability to make anonymous contributions and the absence of face-to-face contact were both singled out for positive comment: for instance, one English student said she had felt less intimidated by being able to discuss the texts without having to betray her lack of knowledge of correct pronunciations.

The class tutor was disappointed that more students did not use the resource, and that 'it had not really taken off': he had hoped that the students would get as far as beginning to suggest texts themselves, but that did not happen. The lack of feedback from students who did not use the resource left it unclear as to why they had not done so, and the actual impact on the learning of those who did use it was similarly not clear. Despite this, the tutor still saw on-line discussion as particularly appropriate to the goals of the class, and was enthused enough to want to expand the resource by introducing it earlier, and using it to feed into project work. His plan was to bring it back into the class after a year's break, when incoming students were likely to be more experienced with email, and increasingly comfortable using on-line resources; and when more texts would be available on-line.

\section{Case 2: Music}

The Department of Music at Glasgow University makes considerable use of computing equipment for composition, audio processing, notation and word processing, and communication. New students in the Department are given induction courses in both email and word processing. The introduction of online seminars into the class on '20th Century Music' was, however, the first attempt to use CMC as a teaching resource (for a more detailed description, see Duffy et al., 1995). The hope was that on-line seminars would constitute an effective replacement for traditional seminars, and would motivate students to participate more actively in discussion.

There were 40 students in the class in total, made up of 2nd, 3rd and (a few) 4th year undergraduates, some taking a full degree in music, some taking a joint honours Arts MA, and some taking a degree in electronic engineering with music as a major component. Few knew each other well, having come together as a group only for this class. They had varying but limited prior experience of computer use, and none of on-line seminars. 
Weekly lectures covered particular schools in twentieth century music, giving examples and detailing historical roles and relationships. Seminar groups of five students and three tutors (constant through the course) honed in on important issues, say different interpretations of one particular work, or examples from one particular composer. The task followed the traditional pattern where one student presented an on-line paper on the specified topic, and the other group members contributed to an on-line discussion on that paper over a three-week period, at the close of which the original presenter summed up. Exchanges were asynchronous, taking place at any point during the period. The sender of each contribution was always identified. The class tutors monitored and contributed to the discussions, and other lecturers were 'invited in' by the students where a topic seemed appropriate.

The seminars produced some quite sizeable pieces of work and all students made contributions. However, this may partly have reflected the fact that both presentations and contributions to discussions were assessed (constituting 25\% of the total mark for the class). In general, the contribu-tions were longer, better, but also more formal than those made during previous traditional seminars. On-line discussion had encouraged verbal exchanges too, among some students at least. The evidence from student feedback was mixed. Initially, there were suspicions about this mode of working, one student commenting that "if I'd known this was part of the class, I wouldn't have taken it". As time went on reactions became more positive, and the final evaluations (albeit based only on a sample of students) indicated that the majority had enjoyed the experience and been encouraged to make more contact with the subject. A small percentage remained negative.

Amongst the lecturers, the perception was that there had been considerable variation between groups as to how much and how well the resource was used, reflected in differences in both the frequency and length of interactions. Where groups had made greater input, this was also typically accompanied by greater experimentation in how to use the resource, and more evidence of argumentation. Where input was less, the students were unsure what to say, even with prompting. For some groups the fact that individuals did not know each other had definitely caused problems, for example preventing them from chasing others for contributions when the discussion had gone quiet. There was little concern about either the assessment or the criteria employed, but as the whole course was continuously assessed, students would have expected everything they wrote to be graded. The assessment criteria were published in advance.

In the next session, on-line seminars were again offered as an option, with traditional choices of essays, or compositional assignments, as alternatives for a different music history course: Baroque and Early Classical Music. Very few students took up the seminar option. Two possible reasons for this emerged from investigation: a) staff felt that the history period under consideration did not lend itself so well to debate and discussion as the previous year's Twentieth Century Music title; b) seminars, on-line or otherwise, are not an easy option, for staff or students. If offered a choice, the familiar is seen as the least threatening. 


\section{Factors affecting CMC usage}

The differences between the two courses, so far as task demand variables are concerned, will have affected the students perceptions about the activity, in its own right and as a learning resource. Yet there is no way in which these can be distinguished. Making participation in the Scottish Language seminars compulsory would increase frequency of use among the students, but this does not necessarily benefit the students or improve learning outcomes. Allowing anonymity and freedom to participate or not to Music students would defeat the whole object of the activity. They were required to contribute to the seminars as assessed course tasks, which were, effectively, the products of the course. Scottish literature students were invited to participate, and the seminars were intended to support course task performance in the computer based workshops and essay papers on text analysis.

What this discussion highlights is that for evaluation purposes a simple analysis of outcomes is uninformative, and potentially misleading. In part this is because there is a need to know more about process in order to interpret outcomes. Even then, though, such analyses depend on outcomes being the product of known circumstances with relatively well-defined characteristics. When this condition is met it becomes possible to examine outcomes in relation to these characteristics in a systematic fashion. In the two case studies, however, as in much other work on CMC resources, there is complex variation in individual contact with and use of the resources. It is essential to identify the nature of this variation and its sources before thinking seriously about how usage might relate to outcome.

In large measure, this variation in individual contact can be seen as a consequence of the fact that activities using CMC resources are typically loosely defined, the primary requirement being that some form of exchange should take place on a specified theme, without the organisation or content of that exchange being tightly prescribed. This means that how the resource is used (and with what effect) is open to the influence of a host of factors. These will include the apparent characteristics of the resource and the task for any user, how this affects their action, and what feedback this generates. However, the context of usage also becomes a major influence, since it is this which helps define much of how the resource is perceived and used.

It is this relationship between context and resource, how it affects student activity, and how it might change over time, which has particularly occupied attention in these attempts to make sense of the usage of $\mathrm{CMC}$ resources. As yet it is too early to offer any detailed model of the nature of this relationship, but it is possible to identify the types of factor on which it is necessary to gather information in order to begin to build such a model. These would seem to fall into five broad categories:

\section{System characteristics}

CMC differs from face to face communication in many respects (e.g. the lack of eye contact and intonational cues to regulate exchanges, the lack of non-verbal information about others' attitudes to the communicator, and the possibility of reflection before responding). Such characteristics have been argued to have a 
direct impact on the form of electronic communication. Rutter and colleagues (1981), for instance, proposed that the cuelessness inherent in electronic exchanges leads to a greater focus on the task in hand, and a generally depersonalised style of interaction. However, it is probably mistaken to assume that $\mathrm{CMC}$ resources have any global or uniform effect of this kind, not least because system characteristics have the potential to differ in many ways (e.g. the extent to which communication is asynchronous or anonymous, whether it is possible to have private and public comment alongside each other, and so on). It is more plausible that, depending on the specific system characteristics, a given resource will facilitate certain types of exchanges, making these more likely and others less so.

The key concept here is that of 'affordance', first defined in the context of ecological psychology (Gibson, 1966) but applied to CMC and the use of electronic media by Gaver (1992). Affordances are the properties of an object which stand in such relation to the properties of an organism that they readily allow some actions to be performed by that organism, but not others (e.g. the properties of a chair afford the action of sitting to an adult human, but not the action of lying down). Affordances are seen as 'natural' in that they are always more perceptible, and resultant actions are more economically performed, so more likely to occur. In the context of CMC resources, a system with an automatic reply function, for example, might afford greater rapidity (and spontaneity) of response, relative to systems without this functionality.

\section{Rules governing use}

Applying the notion of affordance to CMC usage suggests that the activity of users is directly shaped by the system. The form this takes may be accidental on many occasions (it just happens that the system has certain properties), but sometimes it may be deliberate, if, for instance, system characteristics are chosen to fit a specific task (e.g. a discussion between students, to which tutors have no direct access). This points up, though, that shaping by the system itself will often be overlaid by a social dimension, reflecting decisions taken by people about the task set, who will be involved, and most importantly, the 'rules of engagement' between participants (i.e. what kinds of activity are legitimate, and how these should be organised). These rules may be expressed in the first instance via explicit instructions. More commonly, they will be signalled implicitly via activities such as asking questions, thereby providing 'natural' spaces for responses of a particular kind. In this sense, a given resource may in practice offer social as well as physical affordances.

Rules set up in this way may not be static, but may instead evolve over time, as the competences of participants change. Actual extent of engage-ment may also vary as a function of individual differences in how well the rules in operation have been picked up. A common pattern in practice is for initial use of CMC resources to involve direct activity on the part of a limited set of participants, with many more acting as occasional, passive onlookers. This pattern may reflect a process by which usage will eventually increase, termed 'legitimate 
peripheral participation' by Lave \& Wenger (1991). Activity between a small set of more confident participants, which others are allowed to observe from the margins, provides an opportunity for the less engaged to get a picture of the structure of that activity, and what contributions should be like (i.e. the rules in operation). Gradually they may become inducted into the activity themselves, perhaps via requests for responses that they are now capable of making.

\section{Expectations about rules}

In many social contexts, of course, participants will import rules already governing other forms of communication in that context into the use of CMC, rather than developing these from scratch. In particular, HE students typically have considerable experience of the educational system, what activities take place within it, who communicates with whom under what circumstances, and how. CMC usage in this context is therefore extremely likely to be influenced by who students see themselves as communicating with, and what they see as being the root forms of exchange.

One important factor is likely to be whether or not tutors are participants in any discussion: if tutors are involved, they may find it difficult to avoid taking a directive, authoritative role, in part because they are used to this form of communication with students, but also because this is what the students expect, and therefore afford. The result (see e.g. Beattie, 1982) may be a prevalence of formalised exchanges between tutors and individual students (often of limited nature, i.e. tutor-initiation - student-response - tutor-evaluation). These will perhaps be coupled with background exchanges (rather than proper discussion) between the students themselves, if the system allows them to send private messages to each other. It might be possible to set up different communication rules (assuming that one objective of using CMC is to avoid exchanges of the traditional kind) if the tutor deliberately adopts a different role and this is reinforced by an appropriate task (e.g. a joint problem-solving exercise, where it is plain at the outset that the tutor does not possess the solution). Even then, however, students expectations about the tutor's role may be sufficiently ingrained, that traditional exchanges emerge irrespective of what frame the tutor puts on the exercise.

Even in the absence of tutor involvement, student expectations are likely to influence usage. For instance, their communications will be affected by their knowledge of the other individuals involved (or lack of it), and by whether or not they have had some prior training or contact (direct or indirect) with this form of communication, and therefore what they expect to happen when they and others use it. Before they even get to this point, whether or not they use it at all may be influenced by their perception of whether the activity of CMC usage is a legitimate part of their 'contract'. This in turn may be influenced by whether the task involved is assessed, how this task is put to them by the tutor (e.g. whether it is made to seem central or peripheral to other class activity), and even by the physical conditions in which machines are located. 
History

Over time, the experiences that result from the operation of the above factors will themselves become an influence, and usage is likely to become either more refined, or avoided altogether. This may operate in a variety of ways. It may reflect individual learning trajectories: for example, initially students may lack a model for this form of communication and so attempt to map it onto something else (such as essay writing), but discover through use that more spontaneous, informal exchanges are somewhat easier to manage. Conversely, tutors may observe what type of system set up seems to work best for which type of exchange, and modify their resource (and its physical and social affordances) accordingly. The expectations of incoming students may also be shifted via indirect information about the role of CMC resources, and how to use them. This may happen simply through casual observation and listening to the comments of more experienced users by being in the computing lab at the same time. The point here is that students, teachers and resources all have a history that affects individual behaviour.

\section{Connections to other activity}

It has been emphasised above that the broader context of teaching and learning within which CMC takes place may affect its usage, but CMC may in turn affect other teaching and learning, and these effects may then feed back into $\mathrm{CMC}$ exchanges themselves. For instance, increased familiarity with informal exchanges about ideas via CMC might facilitate other classroom discussion, and this may then in turn lead to increased use of CMC for exactly these kinds of exchanges. Similarly, there is evidence (Crook, 1994) that exchanges of ideas between peers have an impact on conceptual grasp which might spill over into other areas of performance in a more fundamental way.

This type of connection between CMC and other activities is of more than passing interest, since the extent to which it occurs will have significant impact on whether CMC activity is perceived to be an integral part of course work. This will have consequences in turn both for the content of exchanges and students' motivation to use the resource. This is not just a matter of tacit connection, however, but of students actually seeing linkages for themselves: activities and learning are often compartmentalised, and there may be sizeable individual differences in making connections of this kind (see e.g. Brown \& Campione, 1988).

\section{Conclusion}

What examination of the usage of CMC in HE points to is the role of cultural as well as cognitive factors, and how usage and culture influence each other over time to produce varying individual responses to the provision of such resources. These factors are without doubt at work affecting more traditional educational resources, but CMC makes them more obvious because it allows much greater scope for individual activity to vary according to perception and experience 
(and also because at present individual differences in experience with computers and $\mathrm{CMC}$ are themselves still considerable).

This perspective in itself cannot be claimed to be especially new (Mantovani, 1996; Spears \& Lea, 1992). As yet, however, not much consideration has been given in the literature as to how in practice this ought to affect evaluation. The implication is that any evaluation of CMC resources should attempt to gather a variety of types of information, and that in general the approach would be to use multiple data sources, mixed methods, and some form of 'triangulation' between these.

Attempts to understand how usage of CMC in HE works have highlighted the influence of cultural factors that had been to some extent hidden in previous research on communication and learning. Once these factors have been identified it has been possible to move on to look at some implications of this shift in perspective for methods and means of inquiry. However, one issue remains open to debate, and this is the question of how comprehensive data collection actually needs to be. Plainly, the need is for collection, coordination and analysis of a considerable quantity of data, and this would involve a large amount of labour. In fact, though, it might not be necessary to be as encyclopedic as this suggests: as long as factors representative of the range are tapped into, it might be possible to build up across projects a 'critical mass' of related data, which would still allow a more complete understanding of the processes involved. The problem is that at this early stage it would also be possible to miss a key piece of information, undermining the usefulness of much other data. The compre-hensive approach might repay rather larger dividends in terms of both understanding CMC usage and devising more effective implementations.

\section{References}

Beattie, G.W. (1982) The dynamics of university tutorial groups. Bulletin of the British Psychological Society, 35, April/4 147-150.

Brown, A.L. \& Campione, J.C. (1986) Psychological theory and the study of learning disabilities. American Psychologist, 14, 10, 1059-1068.

Crook, C. (1994) Computers and the Collaborative Experience of Learning. Routledge, London.

Duffy, C., Arnold, S. \& Henderson, F. (1995) NetSem: Electrifying undergraduate seminars. In Active Learning 2 (July) pp. 42-48. CTISS Publications, Oxford.

Gaver, W.W. (1992) The affordances of media spaces for collaboration. In CSCW'92: Sharing Perspectives - Proceedings of the Conference on Computer Supported Cooperative Work. Toronto.

Gibson, J.J. (1966) The Senses Considered as Perceptual Systems. George Allen \& Unwin, London.

Kaye, A. (1989) Computer-mediated communication and distance education. In Mindweave (eds. R. Mason \& A. Kaye) pp. 3-21. Pergamon Press, Oxford.

Lave, J. \& Wenger, E. (1991) Situated Learning — Legitimate Peripheral Participation. Cambridge University Press, Cambridge.

Mantovani, G. (1996) New Communication Environments. Taylor \& Francis, London.

Rutter, D.R., Stephenson, G.M. \& Dewley, M.E. (1981) Visual communication and the content and style of conversation. British Journal of Social Psychology, 20, 1, 41-52. 